\title{
COVID-19: high-resolution computed tomography findings in the first 64 patients admitted to the Hospital of Cremona, the epicentre of the pandemic in Europe
}

\author{
Vittorio Sabatino $0^{1 A, B, C, D, E, F G^{*}}$, Pietro Sergi ${ }^{1 A, B, C, D, E, F G^{*}}$, Margherita Muri ${ }^{1 B, D}$, llaria Zangrandi ${ }^{1 B, D}$, Giuseppe Voltini ${ }^{1 A, B, D}$, \\ Giancarlo Bosio ${ }^{2 A, D}$, Monia Betti ${ }^{2 A, D}$, Francesca Baglivo ${ }^{2 A, B, D, F}$, Enrico Martinellii ${ }^{2 A, D}$, Angelo Pan ${ }^{3 A, D}$, \\ Matteo Giorgi Pierfranceschi ${ }^{4 A, D}$, Antonio Corvino ${ }^{5 E, F}$, Laura Romanini ${ }^{A, B, B, C, D, E, F, G}$ \\ 'Radiology Department, Hospital of Cremona - Azienda Socio Sanitaria Territoriale di Cremona, Italy \\ 2Pulmonology Department, Hospital of Cremona - Azienda Socio Sanitaria Territoriale di Cremona, Italy \\ ${ }^{3}$ Infectious Diseases Department, Hospital of Cremona - Azienda Socio Sanitaria Territoriale di Cremona, Italy \\ ${ }^{4}$ General Internal Medicine Department, Hospital of Cremona - Azienda Socio Sanitaria Territoriale di Cremona, Italy \\ ${ }^{5}$ Department of Motor Science and Wellness, University of Naples "Parthenope", Italy
}

\section{Abstract}

Purpose: In December 2019, a new coronavirus (SARS-CoV-2) was identified as being responsible for the pulmonary infection called COVID-19. On 21 February 2020, the first autochthonous case of COVID-19 was detected in Italy. Our goal is to report the most common chest computed tomography (CT) findings identified in 64 patients, in the initial phase of COVID-19.

Methods: Sixty-four chest high-resolution computed tomography (HRCT) examinations performed at the Radiology Unit of the Hospital of Cremona, from 22 to 29 February 2020, of 64 patients during first week of hospitalization for COVID-19 were retrospectively evaluated. All cases were confirmed by real-time RT-PCR for SARS-CoV-2. Image analysis was independently conducted by 2 radiologists with 10 years and 1 year of experience in chest imaging. The inter-observer agreement was obtained by applying a Cohen's $\kappa$ test.

Results: The average age of patients was 67.1 years $( \pm 12.2)$; men $42(66 \%)$. HRCT was performed on the $5^{\text {th }}( \pm 1.5)$ day of hospitalization. More frequently, the initial CT changes of the lung show more or less extensive areas of ground-glass, as single pattern or with parenchymal consolidations. Coronavirus lung involvement appears very frequently multi-lobar, bilateral, and it concerns both subpleural and central regions. An excellent agreement ( $\kappa: 0.88-1$, CI: $0.79-1.01, p<0.05$ ) concerning CT findings between the 2 operators was reached.

Conclusions: Our data suggest that detection of the most frequent pulmonary CT-scan changes, in the early stages of COVID-19, can be performed, with excellent agreement, among readers with different experience, and consequently attribute their exact diagnostic value, in an appropriate clinical and environmental exposure setting.

Key words: COVID-19, SARS-CoV-2, coronavirus, HRCT, pulmonary infection, epidemic.

\section{Introduction}

In December 2019, a new coronavirus (SARS-CoV-2) was identified as being responsible for the pulmonary infec- tion called COVID-19 (coronavirus disease 2019) [1-5]. On 21 February 2020, the first autochthonous case of coronavirus infection (COVID-19) was detected in Italy in a 38-year-old man who arrived in an emergency room in

Correspondence address:

Vittorio Sabatino, Radiology Department, Hospital of Cremona - Azienda Socio Sanitaria Territoriale di Cremona, Italy, e-mail: vittorio.sabatino@gmail.com

Authors' contribution:

A Study design · B Data collection - C Statistical analysis · D Data interpretation - E Manuscript preparation · F Literature search · G Funds collection

* Sabatino V. and Sergio P. contributed equally to this work as first authors. 
the Lombardy region in the Hospital of Codogno $(30 \mathrm{~km}$ away from Cremona) with fever and respiratory symptoms.

Approximately 7 days after the first infection, the Hospital of Cremona (Lombardy) was treating about 109 coronavirus patients (2019-nCoV). Symptomatic patients (COVID-19) in almost all cases had non-productive cough, fever, and respiratory failure. In the first week of hospitalization, about 65 patients underwent high-resolution computed tomography (HRCT) of the chest. Chest computed tomography (CT) investigations of the chest were necessary, especially in cases of inconsistency between the patient's clinical data and the findings of the corresponding chest X-rays.

Our aim is to present the most common findings identified in 64 HRCTs of the chest related to 64 different patients with COVID-19, in the initial stage of infection.

\section{Material and methods}

Our institutional review board (IRB) waived written informed consent for this retrospective study, not recognizing any potential risks of violation of patient confidentiality.

Sixty-four chest HRCT examinations, performed at the Radiology Unit of the tertiary Hospital of Cremona, from 22 February to 29 February 2020, in 64 patients during the first week of hospitalization for COVID-19, were retrospectively evaluated. All cases were confirmed by real-time RT-PCR for SARS-CoV-2. The analysis of the chest CT exams was independently conducted by 2 radiologists with 10 years (Reader A) and 1 year (Reader B) of experience in chest imaging.

\section{Table 1. Demographic characteristics}

\begin{tabular}{|l|c|}
\hline Parameter \\
\hline Number of patients & 64 \\
\hline Sex & $M=42(66 \%), F=22(34 \%)$ \\
\hline Age (years) & $67.1( \pm 12.2$ SD; range: $32-85)$ \\
\hline Days* & $5( \pm 1.5$ SD) \\
\hline
\end{tabular}

*Average interval of days between hospitalization and execution of the first chest computed tomography scan
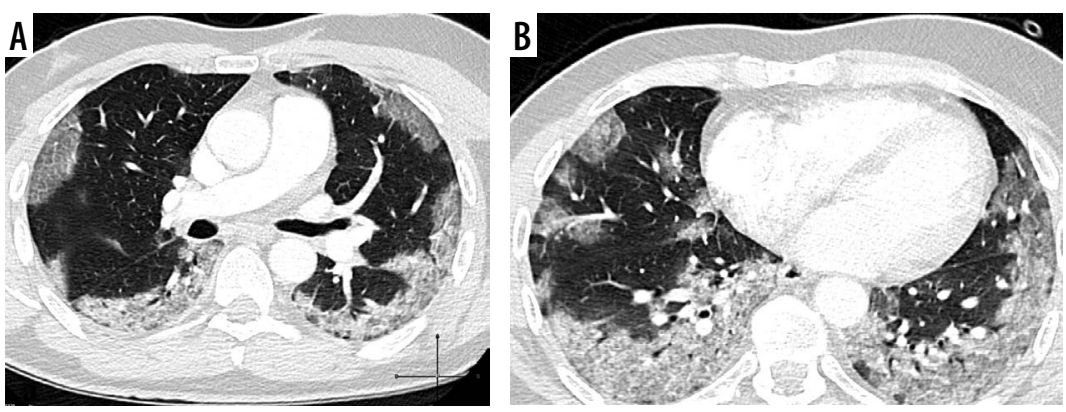

Figure 1. Chest high-resolution computed tomography in a 57-year-old man, unknown comorbidities. Fever and non-productive cough. Leukocytosis. Hypoxaemic and hypocapnic respiratory failure. Suspected contact with red zone subjects. NCOV-19 positive swab. A and B) In all pulmonary lobes extensive areas of increased attenuation are evident. In particular, the prevailing pattern is represented by ground glass opacities, with a tendency towards consolidation in the posterior regions. C) The alterations present a predominantly subpleural distribution and appear more evident at the lower lobes

\section{Results}

A standard nomenclature defined by the Fleischner Society glossary [6] was used for CT findings: crazy paving, ground glass, consolidation, and perilobular pattern. A semi-quantitative scoring system was used to quantitatively estimate the pulmonary involvement. Lung involvement was classified mild, moderate, and severe. Mild involvement was when the sum of the parenchymal abnormalities in both lungs was equal to or less than the volume of the respective upper segment of the Right Upper Lobe (RUL). Moderate involvement was when the sum of the parenchymal abnormalities in both lungs was greater than the volume of the respective upper segment of the RUL, but equal to or less than the overall RUL volume. Severe involvement was if the sum of the parenchymal abnormalities in both lungs was greater than the RUL volume.

The distribution of the findings was recorded as subpleural (involving the peripheral lung zones, including perifissural zones, less than $1 \mathrm{~cm}$ from pleural surface), central (if more than $1 \mathrm{~cm}$ from the pleural surface, including perifissural zones), or combined (when subpleural and central lesions coexisted). The number of lung lobes involved was recorded: right upper lobe (RUL), left upper lobe (LUL), right middle lobe (RML), right lower lobe (RLL), and left lower lobe (LLL), and we considered lingula as different lobes for better classification. The presence or absence of pleural effusion was also recorded.

The inter-observer agreement was obtained by applying a Cohen's $\kappa$ test ( 0 : no concordance, 1 : maximum concordance). The results are reported with $95 \%$ confidence interval, and a $p$-value $<0.05$ was considered statistically significant. Data were analysed with Statistical Analysis System (IBM SPSS Statistics v. 23).

The average age of patients undergoing HRCT of the chest was $67 \pm 12$ years (range: $32-85$ years). Men totalled $42(66 \%)$ and women $22(34 \%)$. On average the CT scan examination was performed on the $5^{\text {th }} \pm 1.5$ day of hospitalization (Table 1). 

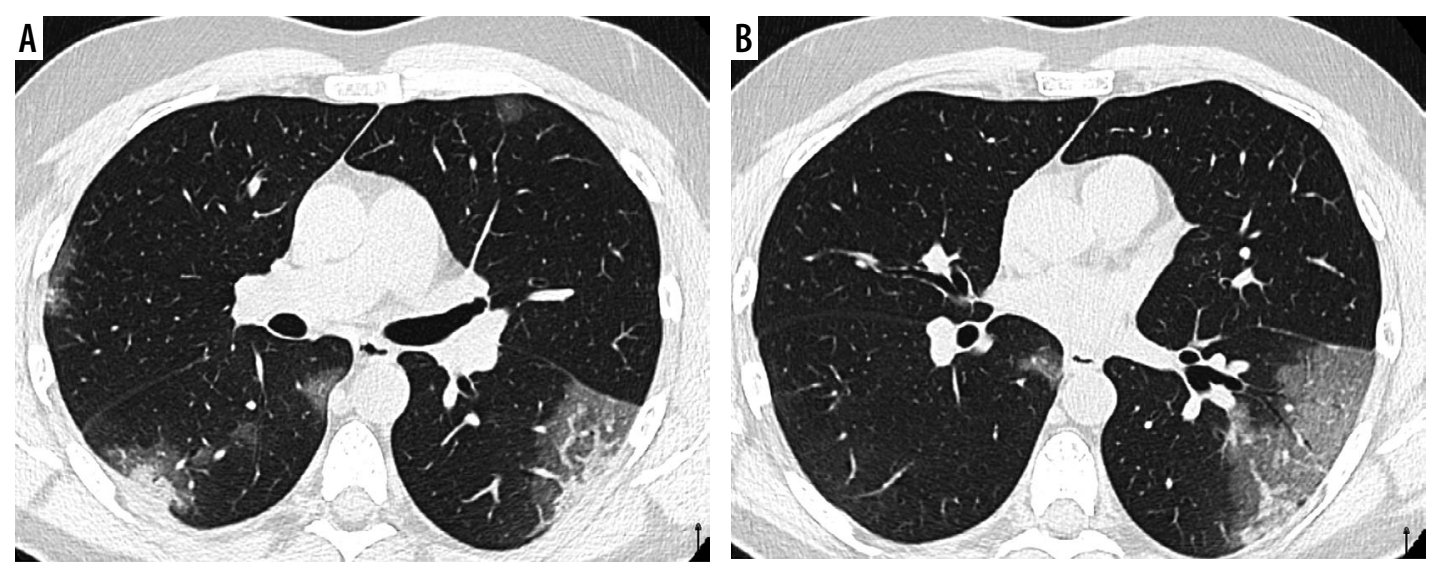

Figure 2. Chest high-resolution computed tomography in a 43-year-old man, unknown comorbidities. Fever and non-productive cough. Leukopaenia. Hypoxaemic and hypocapnic respiratory failure. Contact with red zone subjects. NCOV-19 positive swab. Extensive ground glass opacity in the left lower lobe, with consolidation in the context (mixed lesion). Smaller lesion with similar attenuation features in the right lower lobe. Small ground glass areas in both upper lobes. The alterations have a subpleural distribution

\section{Data relating to Reader A}

Ground glass opacities were identified in 63 patients (98.4\%), parenchymal consolidations in 38 patients (59.3\%), perilobular pattern in 17 patients $(26.5 \%)$, and crazy paving pattern in 4 patients $(6.2 \%)$. The most frequent association between the different patterns was the combination of ground glass and consolidation in 37 patients (57.8\%) (Figures 1 and 2). On average 5.3 lobes per patient were involved. Central distribution of the alterations was identified in 50 patients $(78.1 \%)$, subpleural distribution in 58 patients (90.6\%), and the combination of central and subpleural distribution in 44 patients (68.7\%) (Figure 3). The extension was judged mild in 15 patients $(23.4 \%)$, moderate in 22 patients $(34.3 \%)$, and severe in 27 patients (42.1\%). Pleural effusion was identified in 7 patients.

\section{Data relating to Reader B}

Ground glass opacities were identified in 62 patients (96.8\%), parenchymal consolidations in 39 patients
(60.9\%), perilobular pattern in 12 patients $(18.7 \%)$, and crazy paving pattern in 6 patients $(9.3 \%)$. The most frequent association between the different patterns was the combination of ground glass and consolidation in 37 patients (57.8\%). On average 5.4 lobes per patient were involved. Central distribution of the alterations was identified in 45 patients $(70.3 \%)$, subpleural distribution in 62 patients $(96.8 \%)$, and the combination of central and subpleural distribution in 43 patients $(67.1 \%)$. The extent was judged mild in 10 patients $(15.6 \%)$, moderate in 20 patients $(31.2 \%)$, and severe in 34 patients $(53.1 \%)$. Pleural effusion was identified in 7 patients (Table 2).

An excellent agreement (Cohen's $\kappa: 0.88-1$, CI: 0.79-1.01, $p<0.05$ ) was found between the 2 operators concerning CT findings (Table 3).

\section{Discussion}

Numerous experiences from endemic areas of China suggest that chest CT scan is a vital tool in the diagnostic algorithm for patients with suspected COVID-19 infection
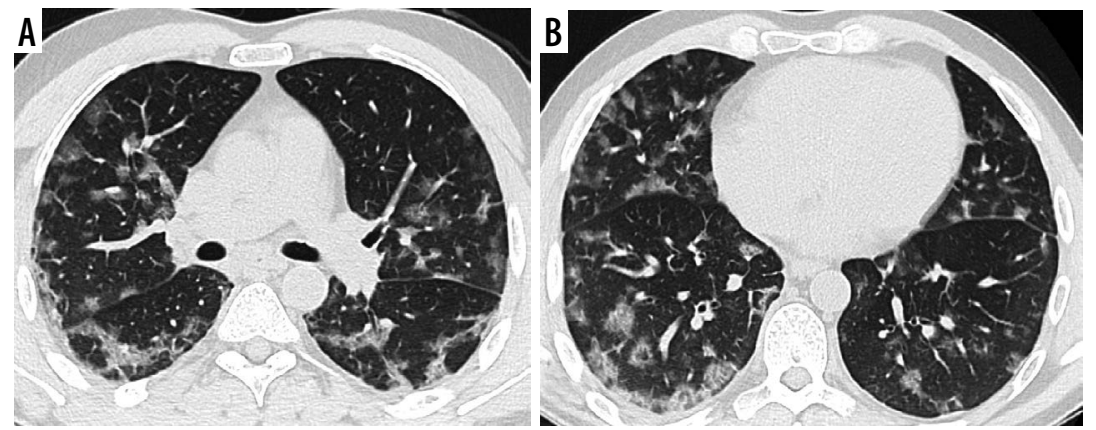

Figure 3. Chest high-resolution computed tomography in a 45-year-old man, unknown comorbidities. Fever and non-productive cough. Leukocytosis. Hypoxaemic and hypocapnic respiratory failure. Contact with red zone subjects. NCOV-19 positive swab. A and B) In all pulmonary lobes there are evident multiple areas of ground glass attenuation. In the subpleural regions of the apical segments of both lower lobes, a perilobular distribution of ground-glass lesions is observed. C) The ground glass areas have both central and subpleural distribution

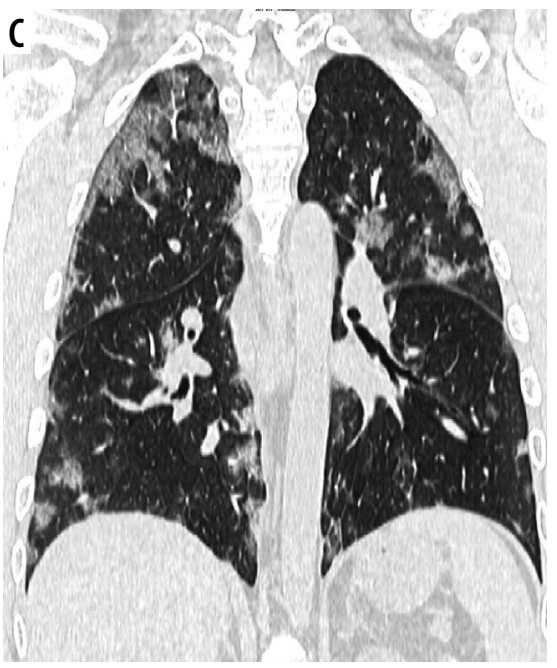


Table 2. High-resolution computed tomography (HRCT) lung findings

\begin{tabular}{|c|c|c|}
\hline HRCT findings & Reader A & Reader B \\
\hline \multicolumn{3}{|l|}{ Pattern } \\
\hline Ground-glass & $63 / 64(98.4 \%)$ & $62 / 64(96.8 \%)$ \\
\hline Consolidation & $38 / 64(59.4 \%)$ & $39 / 64(60.9 \%)$ \\
\hline Perilobular & $17 / 64(26.6 \%)$ & $12 / 64(18.7 \%)$ \\
\hline Crazy paving & $4 / 64(6.2 \%)$ & $6 / 64(9.4 \%)$ \\
\hline Ground-glass and consolidation & $37 / 64(57.8 \%)$ & $37 / 64(57.8 \%)$ \\
\hline \multicolumn{3}{|l|}{ Distribution } \\
\hline Central & $50 / 64(78.1 \%)$ & $45 / 64(70.3 \%)$ \\
\hline Subpleural & $58 / 64(90.6 \%)$ & $62 / 64(96.8 \%)$ \\
\hline Combined & $44 / 64(68.7 \%)$ & $43 / 64(67.1 \%)$ \\
\hline \multicolumn{3}{|l|}{ Localization } \\
\hline Number of involved lobes & $5,3 / 6(88.3 \%)$ & $5,4 / 6(90.0 \%)$ \\
\hline \multicolumn{3}{|l|}{ Lung involvement } \\
\hline Mild & $15 / 64(23.4 \%)$ & $10 / 64(15.6 \%)$ \\
\hline Moderate & $22 / 64(34.3 \%)$ & 20/64 (31.1\%) \\
\hline Severe & $27 / 64(42.2 \%)$ & $34 / 64(53.1 \%)$ \\
\hline Pleural effusion & $7 / 64(10.9 \%)$ & $7 / 64(10.9 \%)$ \\
\hline
\end{tabular}

[4,5,7-10]. In particular, it is useful as a preliminary study in the evaluation of pulmonary abnormalities, in defining the extent of the disease, and in assessing the subsequent course of the disease. Non-contrast high-resolution computed tomography is the method of choice in patients with suspected or confirmed COVID-19 [11]. Integrated imaging for COVID-19 is the best key to reaching a confident diagnosis [12].

In our Radiology Department, during the first week of hospitalization, 64 symptomatic patients with COVID-19 infection underwent HRCT of the chest. Fifty-eight patients out of 64 had simultaneous fever, cough, and respiratory failure. Chest CT-scan investigations were necessary, especially in cases of inconsistency between the patient's clinical data and the findings of the corresponding chest $\mathrm{X}$-rays. These data were due to the low sensitivity of the chest X-ray in the detection of interstitial alterations (e.g. ground-glass), and the rapid progression of pulmonary parenchymal findings.

The incidence of COVID-19 appears to be more frequent in the male population (Table 1), as described by Sun et al. [13]. Moreover, our data allow us to state that coronavirus lung involvement (COVID-19) is very frequently multi-lobar, bilateral, and affects both the subpleural and central regions of the lungs. Subpleural involvement is moderately more frequent than central involvement (Table 2).

More frequently, the initial CT findings of the lung are represented by more or less extensive areas of ground glass as a single pattern or in association with parenchymal consolidations. Infrequent patterns are represented by
Table 3. Inter-observer agreement between first and second operator. Kap$\mathrm{pa}-$ Cohen's $\kappa, \mathrm{Cl}-$ confidence intervals. $P$ - statistical significance $<0.05$

\begin{tabular}{|l|c|c|}
\hline Inter-observer agreement & Kappa & $95 \% \mathrm{Cl}$ \\
\hline Pattern & & \\
\hline Ground-glass & 0.99 & $0.97-1.01$ \\
\hline Consolidation & 0.87 & $0.80-0.93$ \\
\hline Perilobular & 0.86 & $0.79-0.92$ \\
\hline Crazy paving & 0.99 & $0.97-1.01$ \\
\hline Distribution & 0.88 & $0.82-0.94$ \\
\hline Lung involvement & 0.88 & $0.82-0.94$ \\
\hline Pleural effusion & 1 & 1 \\
\hline
\end{tabular}

crazy paving and peri-lobular changes. Such patterns, in our experience, are always in association with ground glass areas and/or consolidations, and never dominant pattern. The parenchymal consolidations and ground-glass areas may also show areas of sparing lobular morphology, and their zonal arrangement does not respect a sub-segmental, segmental, or lobar anatomical distribution.

With regard to the nature of the highlighted lesions, we interpret the findings as diffuse alveolar damage (DAD) or acute fibrinous and organizing pneumonia (AFOP) alterations. We prefer these terms in the radiological description, rather than ARDS, considering ARDS as the clinical syndrome associated with such alterations [14].

We can add that no alterations such as centrilobular nodules, tree in bud patterns, or alterations of the peripheral airways, typical data of inflammatory involvement of small airways, of bronchiolitis and/or parenchymal type (e.g. acinar), were found. There was infrequent evidence of pleural effusion, and when present, it was commonly bilateral and mild.

A further element of interest is the degree of pulmonary involvement in the early stages of the disease. More specifically, we have identified a semi-quantitative criterion of lung disease extension, in addition to clinical signs and laboratory parameters, for the risk stratification of patients with COVID-19. In particular, in the management of our patients, it was important to know that in the initial phase of the disease about $23 \%$ of the subjects had mild pulmonary involvement and about $42 \%$ of our patients had severe pulmonary involvement (Table 2 ). We need a further period of observation and further investigation to understand whether the degree of initial extension of the disease can be considered a prognostic factor in the subsequent clinical course of the disease.

It is interesting to observe that for all the data collected by the two different operators, with different degrees of experience in thoracic radiology (10 years and 1 year, respectively), the inter-observer concordance between the various CT alterations was always in an optimal range $(\kappa$ 0.8-1). In our opinion, that shows that the main identified CT characteristics of COVID 19 can be easily diagnosed by experienced radiologists and by less experienced radiolo- 
gists, making HRCT not only sensitive but also relatively objective.

We are aware that our sample is too small to make exhaustive judgements. Moreover, among the descriptive limits of our experience it must be underlined that a mixed ground glass-consolidation pattern was recorded in the same way as a case where a ground glass area and a consolidation area were in 2 distinct lung areas. Furthermore, multiple and focal alterations, distributed peripherally and centrally, were recorded in the same way as a diffuse extension of disease (e.g. lobar). Finally, when present, atelectasis areas were recorded as consolidations.

\section{Conclusions}

We report the data from our experience to show the most common pulmonary HRCT patterns of COVID-19 presentation at an early stage. In addition, we would like to emphasize how the detection of such patterns can be performed with excellent agreement by readers with different experience, and thus assign their exact diagnostic value in an appropriate clinical and environmental exposure setting.

\section{Conflict of interest}

The authors report no conflict of interest.

\section{References}

1. Zhu N, Zhang D, Wang W, et al. A novel coronavirus from patients with pneumonia in China, 2019. N Engl J Med 2020; 382: 727-733.

2. Pan Y, Guan H, Zhou S, et al. Initial CT findings and temporal changes in patients with the novel coronavirus pneumonia (2019$\mathrm{nCoV}$ ): a study of 63 patients in Wuhan, China. Eur Radiol 2020; 30: 3306-3309.

3. Chen N, Zhou M, Dong X, et al. Epidemiological and clinical characteristics of 99 cases of 2019 novel coronavirus pneumonia in Wuhan, China: a descriptive study. Lancet 2020; 395: 507-513.

4. Shi H, Han X, Jiang N, et al. Radiological findings from 81 patients with COVID-19 pneumonia in Wuhan, China: a descriptive study. Lancet Infect Dis 2020; 20: 425-434.

5. Yang W, Cao Q, Qin L, et al. Clinical characteristics and imaging manifestations of the 2019 novel coronavirus disease (COVID-19): a multi-center study in Wenzhou city, Zhejiang, China. J Infect 2020; 80: 388-393.

6. Hansell DM, Bankier AA, MacMahon H, et al. Fleischner Society: glossary of terms for thoracic imaging. Radiology 2008; 246: 697-722.

7. Bernheim A, Mei X, Huang M, et al. Chest CT findings in coronavirus disease-19 (COVID-19): relationship to duration of infection radiology. Radiology 2020; 295: 200463.
8. Yang W, Yan F. Patients with RT-PCR confirmed COVID-19 and normal chest CT. Radiology 2020; 295: E3. doi: 10.1148/radiol. 2020200702.

9. Zu ZY, Jiang MD, Xu PP, et al. Coronavirus disease 2019 (COVID-19): a perspective from China. Radiology 2020; 296: E15-E25.

10. Pan F, Ye T, Sun P, et al. Time course of lung changes on chest CT during recovery from 2019 novel coronavirus (COVID-19) pneumonia. Radiology 2020; 295: 715-721.

11. Cieszanowski A, Czekajska E, Giżycka B, et al. Management of patients with COVID-19 in radiology departments, and indications regarding imaging studies - recommendations of the Polish Medical Society of Radiology. Pol J Radiol 2020; 85: e209-e214.

12. Trovato P, Simonetti I, Rinaldo C, et al. S. COVID-19 integrated imaging: our experience and literature review. Pol J Radiol 2021; 86: e78-e86.

13. Sun P, Lu X, Xu C, et al. Understanding of COVID-19 based on current evidence. J Med Virol 2020; 92: 548-551.

14. Beasley MB. The pathologist's approach to acute lung injury. Arch Pathol Lab Med 2010; 134: 719-727. 